\title{
Current helicity constraints in solar dynamo models
}

\section{Sokoloff ${ }^{1}$, H. Zhang ${ }^{2}$, D. Moss ${ }^{3}$, N. Kleeorin ${ }^{4}$, K. Kuzanyan ${ }^{5}$, I. Rogachevski ${ }^{6}, \mathbf{Y u} \mathbf{G a o}^{2}$ and H. $\mathrm{Xu}^{2}$}

${ }^{1}$ Department of Physics, Moscow State University, Moscow 119992, Russia email: sokoloff.dd@gmail.com

${ }^{2}$ National Astronomical Observatories, Chinese Academy of Sciences, Beijing 100012, China email: hzhang@bao.ac.cn

${ }^{3}$ School of Mathematics, University of Manchester, Manchester M13 9PL, UK email: moss@maths.man.ac.uk

${ }^{4}$ Department of Mechanical Engineering, Ben-Gurion University of Negev, POB 653, 84105 Beer-Sheva, Israel email: nat@menix.bgu.ac.il

${ }^{5}$ IZMIRAN, Troitsk, Moscow Region 142190, Russia email: kuzanyan@izmiran.ru

${ }^{6}$ Department of Mechanical Engineering, Ben-Gurion University of Negev, POB 653, 84105 Beer-Sheva, Israel email: gary@bgu.ac.il

\begin{abstract}
We investigate to what extent the current helicity distribution observed in solar active regions is compatible with solar dynamo models. We use an advanced $2 \mathrm{D}$ mean-field dynamo model with dynamo action largely concentrated near the bottom of the convective zone, and dynamo saturation based on the evolution of the magnetic helicity and algebraic quenching. For comparison, we also studied a more basic 2D mean-field dynamo model with simple algebraic alpha quenching only. Using these numerical models we obtain butterfly diagrams for both the small-scale current helicity and the large-scale magnetic helicity, and compare them with the butterfly diagram for the current helicity in active regions obtained from observations. This comparison shows that the current helicity of active regions, as estimated by $-\mathbf{A} \cdot \mathbf{B}$ evaluated at the depth from which the active region arises, resembles the observational data much better than the small-scale current helicity calculated directly from the helicity evolution equation. Here $\mathbf{B}$ and $\mathbf{A}$ are respectively the dynamo generated mean magnetic field and its vector potential.
\end{abstract}

Keywords. Sun: magnetic fields - dynamo - interior - surface activity - sunspots

\section{Introduction}

The solar activity cycle is believed to be a manifestation of dynamo action somewhere in solar interior which generates waves of quasi-stationary magnetic field propagating from middle latitudes towards the solar equator ("dynamo waves"). The traditional explanation of this dynamo action (Parker, 1955) is based on the joint action of differential rotation and mirror asymmetric convection which results in what has come to be known as the $\alpha$-effect, based on the helicity of the hydrodynamic convective flow. This explanation is however not the only one currently discussed in the literature and, for example, meridional circulation is also suggested as an important co-factor of the $\alpha$-effect, see e.g., Dikpati and Gilman, (2001); Choudhuri et al., (2004).

In turn, traditional dynamo scenarios based on differential rotation and the classical $\alpha$-effect have to include a dynamo saturation mechanism. One of the most popular 
saturation mechanisms is based on a contribution to the $\alpha$-effect from magnetic fluctuations (Pouquet et al., 1976). A relevant quantification of this effect involves considerations of magnetic helicity evolution(e.g., Kleeorin et al., 1995; 2003). Again, this scenario is not the only one that has been suggested: for example Brandenburg (2007) considers coronal-mass ejections as an important part of nonlinear suppression of the dynamo, and Mitra et al. (2011) consider the effects of the solar wind.

A natural way to resolve such controversies is to determine relevant quantities such as the $\alpha$-effect through observations, thus, providing a check on the various scenarios. Such an option is now becoming realistic, starting from the 1990s when the first attempts to observe current helicity in solar active regions have been undertaken (Seehafer, 1990; Pevtsov et al., 1994; Bao and Zhang, 1998; Hagino and Sakurai, 2004).

Twenty years of continuous efforts by several observational groups, with the most systematic contribution coming from the Huairou Solar Observing Station of China, have resulted (Zhang et al., 2010) in reconstruction of the current helicity time-latitude (butterfly) diagram for one full solar magnetic cycle (1988-2005). From this butterfly diagram it is apparent that the current helicity is involved in the solar activity cycle and follows a polarity law comparable with the Hale polarity law for sunspots - but rather more complicated. In other words, the dynamo generated magnetic field is indeed mirror asymmetric, and this mirror asymmetry is involved in the solar activity cycle, and can be used to understand its nature (Kleeorin et al., 2003, Zhang et al., 2006).

The natural step now is to compare the observed current helicity butterfly diagrams with predictions of particular dynamo models of the solar activity cycle. We performed such a comparison for a dynamo model in which dynamo action is more effective in the deeper layers of the solar convective zone simply because the effect is just from the $\Omega$ gradients being larger there (Zhang et al., 2012). Here we summarize our findings in the context of solar dynamos avoiding technical details.

\section{The observed current helicity butterfly diagram and dynamo models}

The general structure of the observed current helicity butterfly diagram for the last two solar cycles can be described as follows. Current helicity is involved in the solar activity cycle and follows a polarity rule comparable to (however, more complicated than) the polarity rule for toroidal magnetic field, which in turn comes from the Hale polarity rule for sunspot groups. Migration of the helicity pattern is clearly visible and located near the toroidal field pattern. The wings of the helicity butterflies are slightly more inclined to the equator than the magnetic field wings, but the former follow in general the latter. Though the current helicity as a quantity is not strictly speaking quadratic in magnetic field, in one the same hemisphere it has has the same predominant sign for both consequently observed solar cycles, with the opposite sign in the other hemisphere (a kind of unchanging dipolar symmetry). There are some domains in the diagram where the current helicity has the reversed sign with respect to the global polarity law. These domains of "wrong" sign are located at the very beginning and the very end of the wings: see Figure 1. This regularity has been observed in available data for both solar cycles 22 and 23 .

Our approach to compare the dynamo models with observations is as follows. We consider two types of dynamo models. Both types of models are 2D mean-field models with an axisymmetric magnetic field that depends on solar radius $r$ and polar angle $\theta$. The third (azimuthal) coordinate is $\phi$, and $\partial / \partial \phi=0$. The dynamo action is based on 


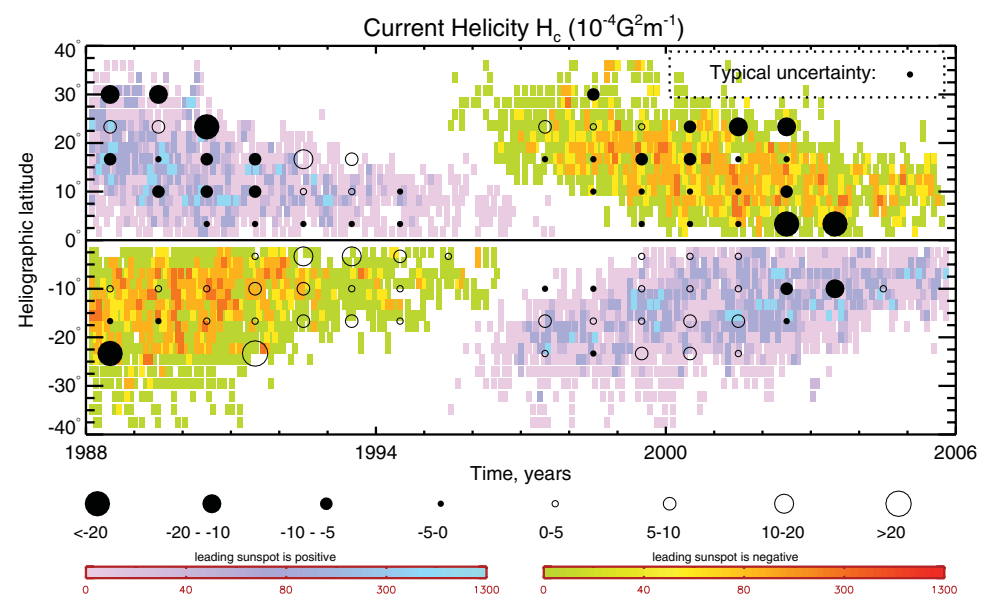

Figure 1. Observed current helicity (white/black circles for positive/negative values) for solar active regions in the $22^{\text {nd }}$ and $23^{\text {rd }}$ solar cycles as averaged over two-year running windows over latitudinal bins of 7 degrees wide, overlaid with sunspot density. The circle in the upper right corner of the panel indicates the typical value of observational uncertainty defined by $95 \%$ confidence intervals scaled to the same units as the circles. 72 out of 88 groups ( $82 \%$ ) have error bars that are smaller than the signal level. The vertical axis gives the latitude in degrees and the horizontal gives the time in years. A colour version of this Figure appears in Zhang et al., (2012).

differential rotation, with a rotation curve which resembles that of the solar convection zone, as known from helioseismological observations, and there is a conventional $\alpha$-effect.

The first type of models assumes a very naive algebraic $\alpha$-quenching. Then we suppose that the total magnetic helicity is locally vanishing, so the magnetic helicity of the large-scale magnetic field produced in the course of mean-field dynamo action has to be compensated by small-scale magnetic helicity. (Thus, we assume that at initial instant the medium is non-magnetic, so that helicity conservation means that the sum of large and small-scale helicities remains locally zero.) We assume also that there is a separation of scales, so that characteristic turbulence scales are much smaller than the characteristic spatial scales of mean magnetic field variations. This allows us to link current and magnetic helicities. This concept underlies the observational procedure for determining the current helicity of active regions, and for calculating the current helicity from the magnetic helicity of the small-scale fields. Based on the same concept we estimate the large-scale magnetic helicity as $B_{\phi} A_{\phi}$, where $A(r, \theta) \hat{\phi}$ is the magnetic potential for the poloidal field. As a result we obtain (for a given radius $r$ ) a theoretical model for the current helicity as a function of $t$ and $\theta$ which we overlay on the butterfly diagram for $B_{\phi}$. We compare the result with the current helicity butterfly diagram known from observations and obtained using similar underlying concepts.

We do not consider this primitive scheme as absolutely realistic. We are sure that any more or less realistic scenario for solar dynamo suppression have to be much more sophisticated. On the other hand, we see that this primitive model produces a helicity butterfly diagram that is quite similar to that observed. The only shortcoming of the model is that the maximum current helicity occurs later than the maximum of $B_{\phi}$, while it is observed to occur probably earlier, though the amount of data available is probably too little to strongly support this statement.

The second type of dynamo models with dynamic suppression of the $\alpha$ - effect is based on helicity helicity conservation principle. If magnetic helicity conservation determines 
the nonlinear dynamo suppression, we expect that a careful reproduction of this balance, including helicity fluxes and the link between magnetic helicity and $\alpha$-effect, will result in even a better theoretical butterfly diagram, and possibly improve the phase relations between helicity and toroidal magnetic field.

As a specific model that takes into account the influence of magnetic helicity balance on dynamo action we use the dynamo model with dynamo action occurring most strongly near the bottom of the convective zone (the model is described in detail by Zhang et al., (2006)). Whereas simple $\alpha$-quenching provides a quite robust suppression of a spherical dynamo and gives (more-or-less) steady nonlinear magnetic field oscillations for a very wide range of parameters, in contrast it is far from clear a priori that a dynamo suppression based on magnetic helicity conservation is effective enough to suppress magnetic field growth and result in steady oscillations. In fact it works more-or-less satisfactorily only in a quite narrow parameter range, which appears inadequate to fit observations convincingly.

We note two crucial points here. First of all, both types of models ignore any direct action of magnetic force on the rotation law. In the more primitive models, there is a crude parametrization of feedback onto the (purely hydrodynamic) alpha effect. The latter, formally more sophisticated model, describes the back-reaction of the generated magnetic field on the dynamo process in terms of the magnetic contribution of the current helicity onto the magnetic part of the $\alpha$-effect. On the other hand, the feedback of the generated large-scale magnetic field on turbulent convection is described in our model by the algebraic quenching of the $\alpha$-effect, turbulent pumping and turbulent magnetic diffusion.

We assume that helicity conservation is not the only mechanism of dynamo suppression. The fact that we see a manifestation of helicity on the solar surface tells us that the buoyancy must play some role, and we add it to the model. We stress that the buoyancy which we include in the model transports current helicity and magnetic helicity as well as large-scale magnetic field.

\section{Results}

We performed an extensive numerical investigation of the models in a parameter range considered to be relevant to solar dynamos.

For the primitive model we found that the simulated current helicity butterfly diagram the plots successfully represent the main features of the observed helicity patterns. Of course, it is possible to choose a set of dynamo governing parameters which may be less similar to those used to describe the Sun. For example the role of magnetic fields in the deeper layers of the convective zone (say, in the overshoot layer) can be emphasized by adjusting the profile of turbulent diffusivity. This tends to make the helicity wave in the overshoot layer look more like a standing wave, but however keeps the main features of the surface diagram. The highly anharmonic standing patterns of butterfly diagrams that were discussed as a possible option for some stars, see Baliunas et al., (2006), look however to be irrelevant for the solar case.

Of course, the helicity pattern in the butterfly diagram obtained in the models for particular choices of dynamo governing parameters can be slightly different from the observed helicity patterns. Xu et al., (2009) demonstrated that meridional circulation can be used to make the simulated pattern resemble more closely to what observed (see their Figure 2).

Zhang et al. (2012) in numerical simulation produced the same type of plots for models based on helicity conservation, to learn that the more or less pronounced migrating 
pattern is associated with the large-scale magnetic helicity only. Small-scale current helicity displays only relatively weak vacillatory behaviour, see Figure 5 in Zhang et al. (2012). We see that the small-scale current helicity is strongly concentrated in middle latitudes and helicity oscillations which are available in the model are almost invisible on the background of the intensive belt of constant helicity in middle latitudes. We doubt that such oscillations would be observable. We stress that, if this model produces any travelling helicity pattern, it is situated in the deep layers only.

The simple models that we have considered here were not intended to reproduce very fine details of the spatial-time distribution of helicity observable in the form of butterfly diagrams, rather to investigate more generally the possible trends and possibilities. We again note that our modelling of helicity in the solar convective zone and active regions is still too simplified to be able to detect more detailed properties of the helicity dynamics.

\section{Conclusions}

We conclude that, at least in the framework of traditional models of the solar dynamo based on dynamo action associated predominately with the deeper regions of the solar convective zone, the current helicity of the magnetic field in active regions is a tracer of the magnetic helicity of the large-scale magnetic field in the solar interior. We believe that this provides a unique option for tracing this quantity, which is very important for the solar dynamo. According to the observational data (Zhang et al., 2010), the current helicity in active regions is mainly negative in the Northern hemisphere. Numerical models give a negative value for $-\mathbf{A} \cdot \mathbf{B}$ in the surface layer of the convective zone in the Northern hemisphere, see Figures 2, 3 and 4 of Zhang et al. (2012).

Summarizing, we conclude that the current helicity of the magnetic field in active regions is expected to have the opposite sign to $\mathbf{A} \cdot \mathbf{B}$, evaluated at the depth at which the active region originates. Thus, the models presented here are consistent with the interpretation that the mechanism responsible for the sign of the observed helicity operates near the solar surface. The mechanism of formation of the current helicity in active regions still requires further investigation.

D.S and K.K. would like to acknowledge support from Visiting Professorship Programme of Chinese Academy or Sciences 2009J2-12 and NAOC of CAS for hospitality, as well NNSF-RFBR grant 08-02-92211 and Russian grant RFBR 10-02-00960a. D.S. is thankful to the RFBR grant 12-02-00170a. H.Z., Y.G. and H.X. are thankful to the Chinese grants 10921303, 11103037, 41174153 and Chinese Academy of Sciences under grant KJCX2-EW-T07.

\section{References}

Baliunas, S., Frick, P., Moss, D., Popova, E., Sokoloff, D., \& Soon, W. 2006, MNRAS, 365, 181 Bao, S. D. \& Zhang, H. Q. 1998, ApJ, 496, L43

Brandenburg, A. 2007, Highlights of Astronomy, 14, 291

Choudhuri, A. R., Chatterjee, P., \& Nandy, D. 2004, ApJ, 615, L57

Dikpati, M. \& Gilman, P. A. 2001, ApJ, 559, 420

Hagino, M. \& Sakurai, T. 2004, Publ. Astron. Soc. Japan., 56, 831

Kleeorin N., Kuzanyan K., Moss D., Rogachevskii I., Sokoloff D., \& Zhang H. 2003, A\&A, 409, 1097

Kleeorin, N., Rogachevskii, I., \& Ruzmaikin, A. 1995, A\& A, 297, 159

Mitra, D., Moss, D., Tavakol, R., \& Brandenburg, A. 2011, A\& A, 526, A138

Parker, E. 1955, ApJ, 122, 293

Pevtsov, A. A., Canfield, R. C., \& Metcalf, T. R. 1994, ApJ, 425, L117 
Pouquet, A., Frisch, U., \& Leorat, J. 1976, J. Fluid Mech., 77, 321

Seehafer, N. 1990, Solar Phys., 125, 219

Xu, H., Gao, Y, Popova, H. P., Nefedov, S. N., Zhang, H., \& Sokoloff, D. D. 2009, Astron. Rep., 53,160

Zhang, H., Sokoloff, D., Rogachevskii, I., Moss, D., Lamburt, V., Kuzanyan, K., \& Kleeorin, N. 2006, MNRAS, 365, 276

Zhang, H., Moss, D., Kleeorin, N., Kuzanyan, K., Rogachevskii, I., Sokoloff, D., \& Gao, Y., Xu H. 2012, ApJ, 751, 47 